\title{
Trace anomaly for non-relativistic fermions
}

\author{
Roberto Auzzi, ${ }^{a, b}$ Stefano Baiguera ${ }^{c}$ and Giuseppe Nardelli ${ }^{a, d}$ \\ ${ }^{a}$ Dipartimento di Matematica e Fisica, Università Cattolica del Sacro Cuore, \\ Via Musei 41, 25121 Brescia, Italy \\ ${ }^{b}$ INFN - Sezione di Perugia, \\ Via A. Pascoli, 06123 Perugia, Italy \\ ${ }^{c}$ Università degli studi di Milano Bicocca and INFN - Sezione di Milano-Bicocca, \\ Piazza della Scienza 3, 20161, Milano, Italy \\ ${ }^{d}$ TIFPA - INFN, c/o Dipartimento di Fisica, Università di Trento, \\ 38123 Povo TN, Italy \\ E-mail: roberto.auzzi@unicatt.it, giuseppe.nardelli@unicatt.it, \\ s.baiguera@campus.unimib.it
}

ABSTRACT: We study the coupling of a $2+1$ dimensional non-relativistic spin $1 / 2$ fermion to a curved Newton-Cartan geometry, using null reduction from an extra-dimensional relativistic Dirac action in curved spacetime. We analyze Weyl invariance in detail: we show that at the classical level it is preserved in an arbitrary curved background, whereas at the quantum level it is broken by anomalies. We compute the trace anomaly using the Heat Kernel method and we show that the anomaly coefficients $a, c$ are proportional to the relativistic ones for a Dirac fermion in $3+1$ dimensions. As for the previously studied scalar case, these coefficents are proportional to $1 / m$, where $m$ is the non-relativistic mass of the particle.

KeywOrds: Anomalies in Field and String Theories, Renormalization Group, Space-Time Symmetries

ARXIV EPRINT: 1705.02229 


\section{Contents}

1 Introduction 1

2 Null reduction for fermions 3

2.1 Metric and frame fields 3

2.2 Dirac action 6

2.3 Flat space-time 6

2.4 Curved spacetime $\quad 7$

2.5 Gyromagnetic ratio 9

3 Weyl invariance $\quad 9$

4 Heat kernel for fermions $\quad 10$

$\begin{array}{lll}4.1 & \text { General framework } & 10\end{array}$

4.2 The flat case 11

4.3 The curved case 12

$\begin{array}{lll}4.4 & \text { A specific perturbation of flat spacetime } & 13\end{array}$

$\begin{array}{lll}4.5 & \text { Perturbative expansion } & 14\end{array}$

$\begin{array}{lll}\text { 4.5.1 Single insertion } & 15\end{array}$

$\begin{array}{lll}\text { 4.5.2 Double insertion } & 15\end{array}$

$\begin{array}{lll}4.5 .3 & \text { Results } & 16\end{array}$

$\begin{array}{lll}5 & \text { Conclusions } & 17\end{array}$

$\begin{array}{ll}\text { A Sigma and gamma matrices with light-cone indices } & 18\end{array}$

$\begin{array}{ll}\text { B Spin connection } & 19\end{array}$

C Some double insertion contributions to the Heat Kernel 20

\section{Introduction}

Newton-Cartan (NC) geometry was originally proposed as a covariant formulation of Newtonian gravity (see e.g. [1] for a review). In recent times it raised growing interest for applications to condensed matter systems (see e.g. [2-5]), such as fermions at unitarity and quantum Hall effect. The background fields of NC gravity provide a natural set of sources for operators in the energy-momentum tensor multiplet of theories with non-relativistic Schrödinger invariance.

Many theoretical difficulties in dealing with these systems are due to the strong coupling nature of the interaction. Strong coupling may drastically change the infrared (IR) 
degrees of freedom coming from a given ultraviolet (UV) description. Renormalization Group (RG) trajectories may interpolate from weak to strong coupling changing the nature of the physical spectrum and of the degrees of freedom. In relativistic theories there are general results which formalize the intuition that information is lost when coarse graining is implemented from UV to IR, namely Zamolodchikov's $c$-theorem in $d=2[6]$, the $F$ theorem in $d=3[7-9]$ the $a$-theorem in $d=4$ [10-15]. For condensed matter applications, it would be interesting to establish similar results in non-relativistic systems.

With these motivations, in the last years a certain amount of work has been devoted to the study of non-relativistic trace anomalies. In general, trace anomalies can be classified into two classes [16]: type A or B depending if they have non-vanishing or vanishing Weyl variation, respectively. The relevant ones for RG constraints are the type $\mathrm{A}$, such as $c$ in $d=2$ or $a$ in $d=4$ relativistic systems.

In the non-relativistic case, at a fixed point space and time may have different relative scaling, which can be parameterized by the dynamical exponent $z$ :

$$
x^{i} \rightarrow e^{\sigma} x^{i}, \quad t \rightarrow e^{z \sigma} t
$$

Moreover one may distinguish between Schrödinger and Lifshitz systems, whose main difference relies on the presence of Galilean boost invariace. So far, in all the known cases, the Lifshitz trace anomalies (see [17-23]) turn out to be of type B and so they do not give interesting candidates for monotonic quantities. In the Schrödinger case, in $d=2+1$ dimensions and for dynamical exponent $z=2$, if one couples the theory to a curved NC background, it exists a type A anomaly [24]. ${ }^{1}$ The structure of this anomaly is the same as the trace anomaly for $d=4$ relativistic theories, and so it includes a type- $A$ and a type- $B$ part, parameterized by $a$ and $c$ coefficients:

$$
\mathcal{A}=-a E_{4}+c W^{2}+\ldots
$$

In this equation $E_{4}$ and $W^{2}$ are the Euler density and the Weyl tensor squared of the null reduction metric in eq. (2.4); these quantities are completely determined in terms of $2+1$-dimensional NC geometry data. The use of the extra dimension is a formidable trick to conveniently keep track of the Milne boost symmetry. Cohomological analysis and general properties were studied in [21, 24-26]. The first explicit calculation of anomalies for a physical system was performed in [27] with the Heat Kernel (HK) method, for the case of a free scalar. Later this result was confirmed in [28] using Fujikawa approach. ${ }^{2}$ On the other hand, both the calculations in [27, 28] disagree with [29].

Fermions are a fundamental ingredient in Nature; the purpose of this paper is to study conformal invariance and anomalies for a free non-relativistic spin $1 / 2$ fermion coupled to a generic curved Newton-Cartan background, using null reduction from a $3+1$ dimensional relativistic action. First of all, we show that it is possible to couple the fermion to the

\footnotetext{
${ }^{1}$ An interesting subtlety is that, in order to get a type-A anomaly, gravity backgrounds which do not satisfy Frobenius condition must be considered [21, 25].

${ }^{2}$ In [28] an extra term is present, which could not be detected with the background chosen in [27]; this issue deserves further study which goes beyond the purpose of the present paper.
} 
geometry in a Weyl invariant way; this is not trivial, due to the different scaling properties of the components of frame fields, spin connection and dynamical fermionic fields. Our analysis specializes to the case where the gyromagnetic ratio $g$ is twice the spin $s$; the generic case requires modified Milne boost transformations [5] on the sources and can not be studied by null reduction.

The other issue that we address is the computation of the anomaly coefficients $a$ and $c$ using Heat Kernel. In the bosonic case, these coefficients turn out to be proportional to the corresponding ones in relativistic systems in $3+1$ dimensions. We find that the same property still persists also in the fermionic case.

The paper is organized as follows. In section 2 we derive the fermionic action from the null reduction of the Dirac one and we discuss the gyromagnetic factor. In section 3 we show in detail that the action is Weyl invariant. In section 4 we compute the trace anomaly using HK method. We conclude in section 5, tecnical details are in appendices.

\section{$2 \quad$ Null reduction for fermions}

\subsection{Metric and frame fields}

We will consider the coupling of non-relativistic fermions in $2+1$ dimensions to a background $\mathrm{NC}$ geometry. In order to make the implementation of the local version of the Galilean symmetry (Milne boost invariance) more covenient, we use the null-reduction method [30] from an extra-dimensional relativistic $3+1$ dimensional theory. We will sometimes refer to null-reduction method as Discrete Light-Cone Quantization (DLCQ). Useful references about NC geometry include [31-40]. Galilei invariance for fermions was first studied in [41]. For other approaches to couple non-relativistic theories to background NC geometry see [5, 42]. Other applications of null reduction to fermions were discussed in [43].

In our conventions late latin capital indices, like $M, N, \ldots$, correspond to $3+1$ dimensional curved space-time indices, whereas early latin capital indices like $A, B, \ldots$, correspond to tangent space indices, where the metric is locally flat. The coordinate $x^{-}$denotes the null direction of the dimensional reduction. The remaining light-cone coordinate, $x^{+}$, will play the role of time in the lower dimensional non-relativistic theory. Curved space coordinates will be labelled by lower case latin indices $i, j, \ldots$, whereas the tangent space counterparts will be labelled by $a, b, \ldots$. Collectively, space time indices of the lower dimensional theory will be denoted by $\mu, \nu, \ldots$ and $\alpha, \beta, \ldots$ for curved and tangent space coordinates, respectively. Summarizing, DLCQ indices are

$$
\begin{array}{ll}
M=(-, \mu)=(-,+, i) & (i=1,2) \\
A=(-, \alpha)=(-,+, a) & (a=1,2) .
\end{array}
$$

Since the light-cone indices \pm use the same symbols for curved or tangent space indices, we will use the notations

$$
\stackrel{ \pm}{(M)}, \quad \stackrel{ \pm}{(A)}
$$

indicating that they refer to curved (subscript $(M)$ ) or tangent space (subscript $(A)$ ) lightcone coordinates. 
In order to apply the null reduction, we will consider fields of the form

$$
\Psi\left(x^{M}\right)=\psi\left(x^{\mu}\right) e^{i m x^{-}}
$$

and a metric of the form

$$
G_{M N}=\left(\begin{array}{cc}
0 & n_{\nu} \\
n_{\mu} & n_{\mu} A_{\nu}+n_{\nu} A_{\mu}+h_{\mu \nu}
\end{array}\right), \quad G^{M N}=\left(\begin{array}{cc}
A^{2}-2 v \cdot A & v^{\nu}-h^{\nu \sigma} A_{\sigma} \\
v^{\mu}-h^{\mu \sigma} A_{\sigma} & h^{\mu \nu}
\end{array}\right) .
$$

We denote the determinant of the metric as:

$$
\sqrt{g}=\sqrt{-\operatorname{det} G_{A B}}=\sqrt{\operatorname{det}\left(h_{\mu \nu}+n_{\mu} n_{\nu}\right)}
$$

The metric tensor $G_{M N}$ defines a non degenerate $3+1$ dimensional metric whose entrees encode the main ingredients of the $2+1$ dimensional NC geometry: a positive definite symmetric rank 2 tensor $h^{\mu \nu}$, which corresponds to the spatial inverse metric, and a nowhere-vanishing vector $n_{\mu}$ (defining the local time direction), with the condition $n_{\mu} h^{\mu \alpha}=0$. In order to define a spatial metric with lower indices and a connection, one introduces a velocity field $v^{\mu}$, with the condition $n_{\mu} v^{\mu}=1$. Given $\left(h^{\mu \nu}, n_{\mu}, v^{\nu}\right)$, one can then uniquely define $h_{\mu \nu}$, with:

$$
h^{\mu \rho} h_{\rho \nu}=\delta_{\nu}^{\mu}-v^{\mu} n_{\nu} \equiv P_{\nu}^{\mu}, \quad h_{\mu \alpha} v^{\alpha}=0,
$$

where $P_{\nu}^{\mu}$ is the projector onto spatial directions. The velocity vector is not unique (it is only required to satisfy $n_{\mu} v^{\mu}=1$ ) and the ambiguity in the choice of $v$ is related to the last ingredient of the $\mathrm{NC}$ geometry: a non-dynamical gauge field $A_{\mu}$, whose presence is necessary to guarantee Milne boost invariance. This gauge field will act as a source for the particle number symmetry. We introduce, for later convenience, the antisymmetric tensors:

$$
\tilde{F}_{\mu \nu}=\partial_{\mu} n_{\nu}-\partial_{\nu} n_{\mu}, \quad F_{\mu \nu}=\partial_{\mu} A_{\nu}-\partial_{\nu} A_{\mu} .
$$

The null reduction is a useful trick to realise the invariance under the following Milne boost transformations:

$$
\begin{aligned}
v^{\prime \mu} & =v^{\mu}+h^{\mu \nu} \psi_{\nu} \\
h_{\mu \nu}^{\prime} & =h_{\mu \nu}-\left(n_{\mu} P_{\nu}^{\rho}+n_{\nu} P_{\mu}^{\rho}\right) \psi_{\rho}+n_{\mu} n_{\nu} h^{\rho \sigma} \psi_{\rho} \psi_{\sigma}, \\
A_{\mu}^{\prime} & =A_{\mu}+P_{\mu}^{\rho} \psi_{\rho}-\frac{1}{2} n_{\mu} h^{\rho \sigma} \psi_{\rho} \psi_{\sigma},
\end{aligned}
$$

while $n_{\mu}$ and $h^{\mu \nu}$ are invariant. Modified Milne transformation may also be considered, but then the null reduction trick can not be used (see e.g. [31]). The following quantities are Milne invariants but are not $\mathrm{U}(1)$ gauge invariants:

$$
\begin{aligned}
v_{A}^{\mu} & =v^{\mu}-h^{\mu \xi} A_{\xi}, \quad\left(h_{A}\right)_{\mu \nu}=h_{\mu \nu}+A_{\mu} n_{\nu}+A_{\nu} n_{\mu}, \quad \phi_{A}=A^{2}-2 v \cdot A, \\
\left(Q_{A}\right)_{\mu \nu \sigma} & =\left(\partial_{\mu}\left(h_{A}\right)_{\nu \sigma}+\partial_{\nu}\left(h_{A}\right)_{\mu \sigma}-\partial_{\sigma}\left(h_{A}\right)_{\mu \nu}\right),
\end{aligned}
$$

where $A^{2}=h^{\mu \nu} A_{\mu} A_{\nu}$ and $A \cdot v=v^{\mu} A_{\mu}$. Note that the standard NC index notation in $d+1$ dimension does not specify how objects transform under Milne boost, for example $v_{A}^{\mu}$ is invariant and $v^{\mu}$ is not. 
The Levi-Civita connection obtained from the metric in eq. (2.4) is:

$$
\begin{aligned}
\Gamma_{--}^{-} & =\Gamma_{--}^{\mu}=0, \quad \Gamma_{\mu-}^{-}=\frac{1}{2} v_{A}^{\sigma} \tilde{F}_{\mu \sigma}, \quad \Gamma_{\nu-}^{\mu}=\frac{1}{2} h^{\mu \sigma} \tilde{F}_{\nu \sigma}, \\
\Gamma_{\mu \nu}^{-} & =\frac{1}{2}\left(\phi_{A}\left(\partial_{\mu} n_{\nu}+\partial_{\nu} n_{\mu}\right)+v_{A}^{\sigma}\left(Q_{A}\right)_{\mu \nu \sigma}\right), \\
\Gamma_{\nu \rho}^{\mu} & =\frac{1}{2}\left(v_{A}^{\mu}\left(\partial_{\nu} n_{\rho}+\partial_{\rho} n_{\nu}\right)+h^{\mu \sigma}\left(Q_{A}\right)_{\nu \rho \sigma}\right) .
\end{aligned}
$$

One may wonder about the relation between this symmetric connection and $d+1$ dimensional connection $\hat{\Gamma}_{\nu \rho}^{\mu}$ used in the standard torsional formulation of the NC geometry. The latter defines a $d+1$ dimensional covariant derivative $\mathcal{D}_{\mu}$ with the following properties

$$
\mathcal{D}_{\mu} n_{\nu}=0, \quad \mathcal{D}_{\mu} h^{\rho \sigma}=0
$$

and with purely temporal torsion, i.e. $h_{\mu \sigma} \hat{\Gamma}_{[\nu \rho]}^{\sigma}=0$. The connection $\hat{\Gamma}_{\nu \rho}^{\mu}$ (which is not symmetric if $\tilde{F}_{\mu \nu}$ is non-zero) is given by:

$$
\hat{\Gamma}_{\nu \rho}^{\mu}=v^{\mu} \partial_{\rho} n_{\nu}+\frac{1}{2} h^{\mu \sigma}\left(\partial_{\nu} h_{\rho \sigma}+\partial_{\rho} h_{\nu \sigma}-\partial_{\sigma} h_{\nu \rho}\right)+h^{\mu \sigma} n_{(\nu} K_{\rho) \sigma},
$$

where the two form $K_{\mu \nu}$ parametrizes an ambiguity which is not fixed by the conditions in eq. (2.11). The simmetric part $\hat{\Gamma}_{(\nu \rho)}^{\mu}$ is related to $\Gamma_{\nu \rho}^{\mu}$ by the following relation [31]:

$$
\Gamma_{\nu \rho}^{\mu}=\hat{\Gamma}_{(\nu \rho)}^{\mu}+\frac{1}{2} h^{\mu \sigma}\left(Q_{A}\right)_{\nu \rho \sigma},
$$

while $\hat{\Gamma}_{[\nu \rho]}^{\mu}$ is not directly related to $\Gamma_{\nu \rho}^{\mu}$.

Since we are dealing with spinors, the covariant derivative also contains the spin connection term and then it is necessary to introduce an orthonormal frame field (vielbein) which relates the metric in the curved spacetime with the flat tangent space. The metric in the flat tangent space is given by

$$
G_{A B}=G^{A B}=\left(\begin{array}{llll}
0 & 1 & 0 & 0 \\
1 & 0 & 0 & 0 \\
0 & 0 & 1 & 0 \\
0 & 0 & 0 & 1
\end{array}\right)
$$

As usual, the vielbein are defined by the following relations:

$$
\begin{aligned}
G_{M N} & =e^{A}{ }_{M} G_{A B} e^{B}{ }_{N}, & G_{A B} & =e^{M}{ }_{A} G_{M N} e^{N}, \\
e^{A}{ }_{M} e^{M}{ }_{B} & =\delta_{B}^{A}, & e^{M}{ }_{A} e^{A}{ }_{N} & =\delta^{M}{ }_{N} .
\end{aligned}
$$

In order to consider the coupling of fermions to $2+1 \mathrm{NC}$ gravity, the dreibein will be defined by dimensional reduction of fierbein. Such operation is not unique. The following choice turns out to be convenient:

$$
e_{M}^{A}=\left(\begin{array}{c}
e^{-}{ }_{M} \\
e^{+}{ }_{M} \\
e^{a}{ }_{M}
\end{array}\right)=\left(\begin{array}{cc}
e_{-}^{-} & e^{-}{ }_{\mu} \\
e^{+} & e^{+}{ }_{\mu} \\
e^{a}{ }_{-} & e^{a}{ }_{\mu}
\end{array}\right)=\left(\begin{array}{cc}
1 & A_{\mu} \\
0 & n_{\mu} \\
\mathbf{0} & e_{\mu}^{a}
\end{array}\right) .
$$


We can further simplify our expression by using the consistency relations among fielbein with different indices

$$
e^{M}{ }_{A} e^{B}{ }_{M}=\delta_{A}^{B}, \quad e^{A}{ }_{M} e^{N}{ }_{A}=\delta_{M}^{N},
$$

which entail the following constraints:

$$
\begin{aligned}
v^{\mu} e^{a}{ }_{\mu} & =0 & & \left(\text { from } e^{M}{ }_{+} e^{a}{ }_{M}=0\right) \\
n_{\mu} e^{\mu}{ }_{a} & =0 & & \left(\text { from } e^{M}{ }_{a} e^{+}{ }_{M}=0\right) \\
e^{\mu}{ }_{a} e^{b}{ }_{\nu} & =\delta_{a}{ }^{b} & & \left(\text { from } e^{M}{ }_{a} e^{b}{ }_{M}=0\right) \\
h^{\mu \nu} e^{a}{ }_{\mu} e^{b}{ }_{\nu} & =\delta^{a b} & & \left(\text { from } e^{M}{ }_{a} e^{b}{ }_{M}=0\right) \\
e^{a}{ }_{\mu} e^{a}{ }_{\nu} & =h_{\mu \nu}, & e^{\mu}{ }_{a} e^{\nu}{ }_{a}= & h^{\mu \nu}, \quad e^{\mu}{ }_{a}=e^{\mu a}
\end{aligned}
$$

These relations simplify the vielbein with the inverted indices:

$$
e_{A}^{M}=\left(\begin{array}{ccc}
e^{M} & e^{M} & e^{M}
\end{array}\right)=\left(\begin{array}{ccc}
e^{-} & e^{-} & e^{-} \\
e^{\mu} & e^{\mu}{ }_{+} & e^{\mu}{ }_{a}
\end{array}\right)=\left(\begin{array}{ccc}
1 & -v^{\sigma} A_{\sigma} & -h^{\nu \sigma} A_{\sigma} e_{\nu}^{a} \\
0 & v^{\mu} & h^{\mu \nu} e_{\nu}^{a}
\end{array}\right) .
$$

The following relation is useful:

$$
h_{\mu \rho} e_{a}^{\rho}=h_{\mu \rho} h^{\rho \tau} e_{\tau}^{a}=\left(\delta_{\mu}^{\tau}-v^{\tau} n_{\mu}\right) e_{\tau}^{a}=e_{\mu}^{a} .
$$

\subsection{Dirac action}

The Dirac operator is expressed as

$$
\not D=\gamma^{M} D_{M}=\gamma^{A} e^{M}{ }_{A} D_{M}
$$

Conventions for gamma matrices with lightcone indices are summarized in appendix A. The covariant derivative takes the form

$$
D_{M} \Psi=\left(\partial_{M}+\frac{1}{4} \omega_{M A B} \gamma^{A B}\right) \Psi=\left(\partial_{M}+\frac{1}{8} \omega_{M A B}\left[\gamma^{A}, \gamma^{B}\right]\right) \Psi
$$

$\omega_{M A B}$ being the spin connection defined in appendix B. We shall derive the non-relativistic fermion action in 2+1-dimensions from the null reduction of the $3+1$-dimensional Dirac action:

$$
S=\int d^{4} x \sqrt{g} i \bar{\Psi} \not D \Psi
$$

The connection in the covariant derivative $D_{M}$ has no torsion term and so the lagrangian in eq. (2.23) can be made hermitian by partial integration.

\subsection{Flat space-time}

We start by considering the simplest flat case:

$$
n_{\mu}=(1,0,0), \quad h_{\mu \nu}=\operatorname{diag}(0,1,1), \quad v^{\mu}=(1,0,0),
$$


and $A_{\mu}=0$. The Dirac action is just the flat one. The following notation is used:

$$
\Psi\left(x^{M}\right)=\left(\begin{array}{c}
\chi_{L}\left(x^{\mu}\right) \\
\varphi_{L}\left(x^{\mu}\right) \\
\varphi_{R}\left(x^{\mu}\right) \\
\chi_{R}\left(x^{\mu}\right)
\end{array}\right) e^{i m x^{-}} .
$$

and the Dirac Lagrangian can be written as

$$
\begin{aligned}
\mathcal{L}= & -\sqrt{2} m \chi_{L}^{\dagger} \chi_{L}-\sqrt{2} m \chi_{R}^{\dagger} \chi_{R}-\sqrt{2} i \varphi_{L}^{\dagger} \partial_{t} \varphi_{L}-\sqrt{2} i \varphi_{R}^{\dagger} \partial_{t} \varphi_{R}+ \\
& +i \varphi_{L}^{\dagger}\left(\partial_{1}+i \partial_{2}\right) \chi_{L}+i \chi_{L}^{\dagger}\left(\partial_{1}-i \partial_{2}\right) \varphi_{L}-i \chi_{R}^{\dagger}\left(\partial_{1}+i \partial_{2}\right) \varphi_{R}-i \varphi_{R}^{\dagger}\left(\partial_{1}-i \partial_{2}\right) \chi_{R} .
\end{aligned}
$$

We find the Euler-Lagrange equations of motion for the various components:

$$
\begin{aligned}
\chi_{L} & =\frac{i}{m} \frac{1}{\sqrt{2}}\left(\partial_{1}-i \partial_{2}\right) \varphi_{L}, & \chi_{R} & =-\frac{i}{m} \frac{1}{\sqrt{2}}\left(\partial_{1}+i \partial_{2}\right) \varphi_{R}, \\
\partial_{t} \varphi_{L} & =\frac{1}{\sqrt{2}}\left(\partial_{1}+i \partial_{2}\right) \chi_{L}, & \partial_{t} \varphi_{R} & =-\frac{1}{\sqrt{2}}\left(\partial_{1}-i \partial_{2}\right) \chi_{R} .
\end{aligned}
$$

As expected for the Dirac action in the massless case, the left and right Weyl spinors decouple. The auxiliary fields $\chi_{L, R}$ can be eliminated by the equations of motion and replaced in the Lagrangian; we obtain a set of decoupled Schrödinger equations for the fermions $\varphi_{L, R}$.

\subsection{Curved spacetime}

In this section we will write eq. (2.23) in a more explicit way, in order to later establish the gyromagnetic factor and show that the action is conformal invariant. The left and right-handed parts of the Dirac spinor decouple:

$$
\Psi=\left(\begin{array}{c}
\Psi_{L} \\
\Psi_{R}
\end{array}\right), \quad \Psi_{L}=\left(\begin{array}{c}
\chi_{L} \\
\varphi_{L}
\end{array}\right), \quad \Psi_{R}=\left(\begin{array}{c}
\varphi_{R} \\
\chi_{R}
\end{array}\right)
$$

In the remaining part of this section we will consider the action $\mathcal{L}_{1}$ for just the left component $\Psi_{L}\left(\Psi_{R}\right.$ is completely analogous):

$$
\Psi_{L}=\left(\begin{array}{l}
\chi \\
\varphi
\end{array}\right) e^{i m x^{-}}, \quad S_{L}=\int d^{4} x \sqrt{g} \mathcal{L}_{1},
$$

where

$$
\begin{aligned}
\mathcal{L}_{1}=i \Psi_{L}^{\dagger} \bar{\sigma}^{A} D_{A} \Psi_{L}= & i e^{-i m x^{-}}\left(\chi^{\dagger} \varphi^{\dagger}\right) \bar{\sigma}^{-} D_{(\bar{A})}\left[\left(\begin{array}{l}
\chi \\
\varphi
\end{array}\right) e^{i m x^{-}}\right] \\
& +i e^{-i m x^{-}}\left(\chi^{\dagger} \varphi^{\dagger}\right) \bar{\sigma}^{+} D_{(A)}\left[\left(\begin{array}{l}
\chi \\
\varphi
\end{array}\right) e^{i m x^{-}}\right] \\
& +i e^{-i m x^{-}}\left(\chi^{\dagger} \varphi^{\dagger}\right) \sigma^{a} e_{a}^{M} D_{M}\left[\left(\begin{array}{l}
\chi \\
\varphi
\end{array}\right) e^{i m x^{-}}\right] .
\end{aligned}
$$


For convenience, we renamed $\left(\chi_{L}, \varphi_{L}\right)$ as $(\chi, \varphi)$. An explicit calculation gives:

$$
\begin{aligned}
& D_{(\bar{A})}=e_{(\bar{A})}^{M} D_{M}=\left(\begin{array}{ll}
1 & 0
\end{array}\right)\left(\begin{array}{c}
D_{(\bar{M})} \\
D_{\mu}
\end{array}\right)=D_{(\bar{M})}, \\
& \underset{(A)}{D_{+}}=e_{(A)}^{M} D_{M}=\left(-v^{\sigma} A_{\sigma} v^{\mu}\right)\left(\begin{array}{c}
D_{(-)} \\
D_{\mu}
\end{array}\right)=-v^{\sigma} A_{\sigma} D_{(\bar{M})}+v^{\mu} D_{\mu}, \\
& D_{a}=e_{a}^{M} D_{M}=\left(-e^{\sigma}{ }_{a} A_{\sigma} e^{\mu}{ }_{a}\right)\left(\begin{array}{c}
D_{(\bar{M})} \\
D_{\mu}
\end{array}\right)=-e_{a}^{\sigma} A_{\sigma} D_{(\bar{M})}+e^{\mu}{ }_{a} D_{\mu} .
\end{aligned}
$$

We can write $\mathcal{L}_{1}$ as follows:

$$
\begin{aligned}
\mathcal{L}_{1}= & -\sqrt{2} m \chi^{\dagger} \chi-\sqrt{2} i \varphi^{\dagger} \hat{D}_{t} \varphi+i \varphi^{\dagger}\left(\hat{D}_{1}+i \hat{D}_{2}\right) \chi+i \chi^{\dagger}\left(\hat{D}_{1}-i \hat{D}_{2}\right) \varphi \\
& +\frac{i}{4}\left(\chi^{\dagger} \varphi^{\dagger}\right)\left(\bar{\sigma}^{+} v^{\mu}+\sigma^{a} e_{a}^{\mu}\right) \omega_{\mu A B} \sigma^{A B}\left(\begin{array}{c}
\chi \\
\varphi
\end{array}\right) \\
& +\frac{i}{4}\left(\chi^{\dagger} \varphi^{\dagger}\right)\left(\bar{\sigma}^{-}-v^{\sigma} A_{\sigma} \bar{\sigma}^{+}-\sigma^{a} e_{a}^{\sigma} A_{\sigma}\right) \omega_{(M)} A B \sigma^{A B}\left(\begin{array}{c}
\chi \\
\varphi
\end{array}\right),
\end{aligned}
$$

where we introduced derivatives which are covariant with respect to the local $\mathrm{U}(1)$ symmetry:

$$
\hat{D}_{t}=v^{\mu}\left(\partial_{\mu}-i m A_{\mu}\right), \quad \hat{D}_{a}=e^{\mu}{ }_{a}\left(\partial_{\mu}-i m A_{\mu}\right)
$$

The last two lines are more troublesome and require the explicit knowledge of the components of the spin connection, because its Lorentz indices are contracted with sigma matrices, containing also spinorial indices.

We can put the action (2.32) in the following form:

$$
\mathcal{L}_{1}=\left(\begin{array}{ll}
\chi^{\dagger} & \varphi^{\dagger}
\end{array}\right)\left(\begin{array}{ll}
A & B \\
C & E
\end{array}\right)\left(\begin{array}{l}
\chi \\
\varphi
\end{array}\right)
$$

where

$$
\begin{aligned}
& A=-\sqrt{2}\left(m+\frac{1}{4} \tilde{F}_{\mu \nu} e_{1}^{\mu} e_{2}^{\nu}\right), \\
& B=\left(e_{1}^{\mu}-i e_{2}^{\mu}\right)\left(i \tilde{D}_{\mu}+\frac{i}{4} \tilde{F}_{\mu \nu} v^{\nu}\right), \quad C=\left(e_{1}^{\mu}+i e_{2}^{\mu}\right)\left(i \tilde{D}_{\mu}+i \frac{3}{4} \tilde{F}_{\mu \nu} v^{\nu}\right), \\
& E=\sqrt{2}\left[v^{\mu}\left(-i \tilde{D}_{\mu}-\frac{i}{4} h^{\rho \sigma} \partial_{\mu} h_{\rho \sigma}\right)-\frac{i}{2}\left(v^{\mu} v^{\nu} \partial_{\mu} n_{\nu}+\partial_{\mu} v^{\mu}\right)-\frac{1}{4} F_{\mu \nu} e_{1}^{\mu} e_{2}^{\nu}\right] .
\end{aligned}
$$

In these expressions $\tilde{D}_{\mu}$ denotes a partially covariant derivative which includes just the gauge and the curved space spin connection $\tilde{\omega}_{\mu a b}$ built just with the spatial tetrad $e_{\mu}^{a}$; this derivative acts on the matter fields $\varphi$ and $\chi$ as follows:

$$
\tilde{D}_{\mu} \varphi=\left[\partial_{\mu}-\frac{i}{2} \tilde{\omega}_{\mu 12}-i m A_{\mu}\right] \varphi, \quad \tilde{D}_{\mu} \chi=\left[\partial_{\mu}+\frac{i}{2} \tilde{\omega}_{\mu 12}-i m A_{\mu}\right] \chi
$$


where

$$
\tilde{\omega}_{\mu a b}=\frac{1}{2}\left(e^{\nu}{ }_{a}\left(\partial_{\mu} e^{b}{ }_{\nu}-\partial_{\nu} e^{b}{ }_{\mu}\right)-e^{\nu}{ }_{b}\left(\partial_{\mu} e^{a}{ }_{\nu}-\partial_{\nu} e^{a}{ }_{\mu}\right)-e^{\nu}{ }_{a} e^{\rho}{ }_{b} e^{c}{ }_{\mu}\left(\partial_{\nu} e^{c}{ }_{\rho}-\partial_{\rho} e^{c}{ }_{\nu}\right)\right) .
$$

The auxiliary field $\chi$ is determined by the equations of motion as follows:

$$
\chi=\frac{i\left(e_{1}^{\mu}-i e_{2}^{\mu}\right)\left(\tilde{D}_{\mu}+\frac{1}{4} v^{\nu} \tilde{F}_{\mu \nu}\right) \varphi}{\sqrt{2}\left(m+\frac{\tilde{F}_{\mu \nu} e_{1}^{\mu} e_{2}^{\nu}}{4}\right)} .
$$

Replacing it into the action in eq. (2.34), we could obtain a cumbersome Lagrangian written only in terms of $\varphi$. In order to keep our calculations simple, we will later specialize to some specific backgrounds.

\subsection{Gyromagnetic ratio}

Let us compute the gyro-magnetic ratio of the non-relativistic fermion. We consider flat background space-time as in eq. (2.24) and a generic particle number background gauge field $A_{\mu}$. Specializing the general results in appendix B, we find the non-zero components of the spin connection:

$$
\omega_{++i}=-F_{0 i}=-E_{i}, \quad \omega_{i+j}=-\frac{1}{2} F_{i j}=-\frac{B}{2}, \quad \omega_{0 i j}=-\frac{1}{2} F_{i j}=-\frac{B}{2} .
$$

Eliminating $\chi$ with the equations of motion, we obtain:

$$
S=\int d^{3} x\left[\frac{i}{2} \varphi^{\dagger} \stackrel{\leftrightarrow}{\partial}_{t} \varphi-\frac{1}{2 m} \delta^{i j}\left(D_{i} \varphi\right)^{\dagger}\left(D_{j} \varphi\right)-\frac{1}{4} B \varphi^{\dagger} \varphi\right]
$$

In this calculation we considered a left-handed fermion; the case of a right-handed one is completely analogous and gives the same lagrangian, with an opposite sign for the $B \varphi^{\dagger} \varphi$ coupling.

The generic form of the gyromagnetic coupling in $2+1$ dimension is

$$
\mp g \frac{q}{4 m} B \varphi^{\dagger} \varphi
$$

where $q$ is the charge and the $\mp$ sign refers to left or right-handed spinor, respectively. Since in our conventions the charge associated to the particle number symmetry is $q=m$, we find a gyromagnetic ratio $g=1$. This is consistent with the form of the Milne boost transformations which come from null reduction, which are valid for $g=2 s$ [5]: this is the simplest way in which Galilean covariance can be realized.

\section{$3 \quad$ Weyl invariance}

In order to study the conformal symmetry of the theory, it is useful to determine the Weyl weights of the fields appearing in the action. Weyl transformations act on the metric in the following way:

$$
n_{\mu} \rightarrow e^{2 \sigma} n_{\mu}, \quad h_{\mu \nu} \rightarrow e^{2 \sigma} h_{\mu \nu} \quad v^{\mu} \rightarrow e^{-2 \sigma} v^{\mu}, \quad h^{\mu \nu} \rightarrow e^{-2 \sigma} h^{\mu \nu} .
$$


The action on the frame fields is as follows:

$$
\begin{array}{lll}
e_{M}^{-} \rightarrow e_{M}^{-}, & e_{M}^{+} \rightarrow e_{M}^{+} e^{2 \sigma}, & e_{M}^{a} \rightarrow e_{M}^{a} e^{\sigma} \\
e_{-}^{M} \rightarrow e_{-}^{M}, & e_{+}^{M} \rightarrow e_{+}^{M} e^{-2 \sigma}, & e_{a}^{M} \rightarrow e_{a}^{M} e^{-\sigma} .
\end{array}
$$

It is also useful to know how each element of the spin connection transforms under a Weyl transformation:

$$
\begin{array}{lll}
\omega_{-a b} \rightarrow \omega_{-a b}, & \omega_{-+a} \rightarrow e^{-\sigma}\left(\omega_{-+a}+e_{a}^{\nu} \partial_{\nu} \sigma\right), \\
\omega_{\mu-a} \rightarrow e^{\sigma}\left(\omega_{\mu-a}+n_{\mu} e_{a}^{\nu} \partial_{\nu} \sigma\right), & & \\
\omega_{\mu-+} \rightarrow \omega_{\mu-+}-\partial_{\mu} \sigma+n_{\mu} v^{\nu} \partial_{\nu} \sigma, & \omega_{\mu+a} \rightarrow e^{-\sigma}\left(\omega_{\mu+a}+\left(-v^{\nu} e_{\mu}^{a}+e_{a}^{\nu} A_{\mu}\right) \partial_{\nu} \sigma\right), \\
\omega_{\mu a b} \rightarrow \omega_{\mu a b}+\left(e_{\mu}^{a} e_{b}^{\nu}-e_{\mu}^{b} e_{a}^{\nu}\right) \partial_{\nu} \sigma . &
\end{array}
$$

In the usual relativistic case the components of a Dirac spinor have all the same Weyl weight; this is not true in the null reduction setting that we are considering, because the tetrads have different Weyl weights. The transformation of the $(\chi, \varphi)$ components is as follows:

$$
\chi \rightarrow e^{-2 \sigma} \chi, \quad \varphi \rightarrow e^{-\sigma} \varphi .
$$

This can be derived from dimensional analysis in the flat case, see eq. (2.27): in units of length, $[\varphi]=-1$ and $[\chi]=-2$. In the case of a Dirac fermion

$$
\Psi=\left(\begin{array}{c}
\chi_{L} \\
\varphi_{L} \\
\varphi_{R} \\
\chi_{R}
\end{array}\right), \quad \text { dimensions are } \quad[\Psi]=\left(\begin{array}{l}
-2 \\
-1 \\
-1 \\
-2
\end{array}\right)
$$

We note that this Weyl weight choice is crucial in order to assign to the term $\bar{\Psi} \Psi$ a welldefined Weyl weight. A conformal coupling term such as $R \bar{\Psi} \Psi$ would have mass dimension 5 , spoiling conformal invariance.

Promoting $\sigma$ to a spacetime-dependent function in eq. (3.4), one can then verify the Weyl invariance of the action in eq. (2.32) by direct calculation, using the non-homogeneus part of the variation of the spin connection (see eq. (3.3)). One can also check that this is consistent with eq. (2.38): if we insert $\varphi \rightarrow e^{-\sigma} \varphi$, we indeed find that $\chi \rightarrow e^{-2 \sigma} \chi$.

\section{Heat kernel for fermions}

\subsection{General framework}

For a complex field $\phi$, the vacuum functional $W$ is defined by

$$
e^{i W}=\int \mathcal{D} \phi^{\dagger} \mathcal{D} \phi e^{i S_{D}\left[\phi^{\dagger}, \phi\right]}
$$

where $S_{D}$ is the classical action specified by a differential operator $D$. In the bosonic case, the path integral is evaluated in terms of the functional determinant of the operator $D$ as

$$
i W=-\log \operatorname{det}(D)
$$


In the fermionic case there are two differencies: first the change of sign in the r.h.s. of (4.2) due to the Berezin functional integration. Second, there is the difficulty that the Dirac operator $\not D$ is not elliptic after a Wick rotation. This problem can be bypassed by evaluating the determinant of the square of the Dirac operator and inserting a factor $1 / 2$ :

$$
i W=\frac{1}{2} \log \operatorname{det}\left(\not D^{2}\right)
$$

In this way the Euclidean version of the squared Dirac operator is elliptic and meets the requirements needed in order to make the heat kernel computation. In fact, using anticommutation rules for the product of totally antisymmetric Dirac matrices we find (see e.g. $[44,45])$ :

$$
(i \not D)^{2}=-\square+\frac{1}{4} R \equiv-\hat{\triangle}, \quad \square=D_{A} D^{A} .
$$

We need to compute the HK with the imaginary time version of the operator $\widehat{\triangle}$ in eq. (4.4). To this purpose we decompose it as the flat part $\triangle$ plus curved space perturbation $\delta \triangle$ :

$$
\hat{\triangle}=\triangle \mathbf{1}+\delta \triangle, \quad \triangle=\left(-2 i m \partial_{t}+\partial_{i}^{2}\right) .
$$

\subsection{The flat case}

The computation of the HK is performed in imaginary time space. This is realized by the substitutions

$$
t \rightarrow-i t_{E}, \quad \partial_{t} \rightarrow i \partial_{t_{E}}, \quad m \rightarrow i m_{E} .
$$

The HK operator of an operator $\hat{\mathcal{O}}_{E}$ is defined as

$$
\hat{K}_{\hat{\mathcal{O}}_{E}}(s)=\exp \left(s \hat{\mathcal{O}}_{E}\right) .
$$

We will denote by $K_{\hat{\mathcal{O}}_{E}}$ the matrix elements

$$
K_{\hat{\mathcal{O}}_{E}}\left(s, x, t, x^{\prime}, t^{\prime}\right)=\left\langle x t\left|\hat{K}_{\hat{\mathcal{O}}_{E}}(s)\right| x^{\prime} t^{\prime}\right\rangle,
$$

and by $\tilde{K}_{\hat{\mathcal{O}}_{E}}$ the diagonal matrix elements

$$
\tilde{K}_{\hat{\mathcal{O}}_{E}}(s, x, t)=\left\langle x t\left|\hat{K}_{\hat{\mathcal{O}}_{E}}(s)\right| x t\right\rangle .
$$

In the flat non-relativistic case, with operator $\triangle$ in (4.5):

$$
\triangle=\left(-2 i m \partial_{t}+\partial_{i}^{2}\right)=\left(-2 m \sqrt{-\partial_{t}^{2}}+\partial_{i}^{2}\right),
$$

the heat kernel has been evaluated in [27] and its matrix elements read

$$
K_{\triangle}(s)=\left\langle x t\left|e^{s \triangle}\right| x^{\prime} t^{\prime}\right\rangle=\frac{1}{2 \pi} \frac{m s}{m^{2} s^{2}+\frac{\left(t-t^{\prime}\right)^{2}}{4}} \frac{1}{(4 \pi s)^{d / 2}} \exp \left(-\frac{\left(x-x^{\prime}\right)^{2}}{4 s}\right) .
$$

Here a comment is in order: to use the heat kernel machinery with the Schrödinger operator, we use the formal replacement $-2 i m \partial_{t} \longrightarrow-2 m \sqrt{-\partial_{t}^{2}}$. This, by itself, does not render 
the Schrödinger operator elliptic, but it makes possible an integral representation in which the exponential of the Schrödinger operator is written as a sum of exponentials of elliptic operators, which is precisely what is needed to compute the heat kernel, namely

$$
e^{-2 m \sqrt{-\partial_{t}^{2}}}=\int_{0}^{\infty} d \sigma \frac{m}{\sqrt{\pi}} \frac{1}{\sigma^{3 / 2}} e^{-\frac{m^{2}}{\sigma}} e^{-\sigma\left(-\partial_{t}^{2}\right)},
$$

This trick was first introduced in [46], although in a different context, and used in [27] to evaluate the anomaly in the bosonic case. In its essence, this regularization is not different from the one normally used in the relativistic case to adapt the heat kernel procedure to fermions: to make elliptic the Dirac operator, one first considers its square, perform the heat kernel, and then takes the square root of the resulting determinant.

An alternative way to compute the trace anomaly is by the Fujikawa's method: this was recently performed in [28], obtaining the same numerical result for the scalar case. An alternative treatment of the heat kernel procedure, applied to parabolic operators, was developed in [29], obtaining a results which disagrees with both [27] and [28].

\subsection{The curved case}

In order to explicitly compute the functional determinant we work in coordinate representation with scalar product:

$$
\left\langle x t \mid x^{\prime} t^{\prime}\right\rangle_{g}=\frac{\delta\left(x-x^{\prime}\right) \delta\left(t-t^{\prime}\right)}{\sqrt{g}} .
$$

In curved background, the HK can be evaluated as a perturbative expansion around (4.11). The trace of the HK in the coordinate basis can be expanded in powers of $s$ as:

$$
\tilde{K}_{\hat{\triangle}}(s)=\operatorname{Tr}\left\langle x t\left|e^{s \hat{\triangle}}\right| x t\right\rangle_{g}=\frac{1}{s^{d / 2+1}}\left(a_{0}(\hat{\triangle})+a_{2}(\hat{\triangle}) s+a_{4}(\hat{\triangle}) s^{2}+\ldots\right) .
$$

This defines the De Witt-Seeley-Gilkey coefficients $a_{2 k}(\hat{\triangle})$ of the problem. In nonrelativistic $2+1$ dimensional theories, the trace anomaly is proportional to the $a_{4}$ coefficient [27].

It is convenient to introduce a quantum mechanical space, with flat inner product

$$
\left\langle x t \mid x^{\prime} t^{\prime}\right\rangle=\delta\left(x-x^{\prime}\right) \delta\left(t-t^{\prime}\right),
$$

and, for any operator $\hat{\mathcal{O}}$, to define the operator $\hat{M}_{\hat{\mathcal{O}}}$ such that

$$
\left\langle x t|\hat{\mathcal{O}}| x^{\prime} t^{\prime}\right\rangle_{g}=\left\langle x t\left|\hat{M}_{\hat{\mathcal{O}}}\right| x^{\prime} t^{\prime}\right\rangle .
$$

Thus, one introduces and "effective" operator $\hat{M}_{\hat{\mathcal{O}}}$ that keeps track of the metric in the inner product. In our case, if $\hat{\mathcal{O}}=\hat{\triangle}=\square-\frac{1}{4} R$, then

$$
\left\langle x t|\hat{M}| x^{\prime} t^{\prime}\right\rangle=g^{1 / 4}(x, t)\left(\square_{x, t}-\frac{1}{4} R\right)\left[g^{-1 / 4}(x, t) \delta\left(x-x^{\prime}\right) \delta\left(t-t^{\prime}\right)\right] .
$$

In this way we can expand the trace of the HK as

$$
\tilde{K}_{\hat{M}}(s)=\operatorname{Tr}\left\langle x t\left|e^{s \hat{M}}\right| x t\right\rangle=\frac{1}{s^{d / 2+1}}\left[a_{0}(\hat{M})+s a_{2}(\hat{M})+s^{2} a_{4}(\hat{M})+\ldots\right] \equiv \sqrt{g} \tilde{K}_{\hat{\triangle}}(s) .
$$




\subsection{A specific perturbation of flat spacetime}

To proceed, we specialize to a particular perturbation of flat spacetime:

$$
n_{\mu}=\left(\frac{1}{1-\eta\left(x^{i}\right)}, 0,0\right), \quad v^{\mu}=\left(1-\eta\left(x^{i}\right), 0,0\right), \quad h_{i j}=\delta_{i j}, \quad A_{\mu}=0 .
$$

Also we remind that the spatial frame field is a Kronecker delta:

$$
e_{i}^{a}=e_{a}^{i}=\delta_{a}^{i}
$$

For simplicity, we choose $\eta$ independent from the time coordinate. The non-vanishing components of the spin connection and Cristoffel symbols are:

$$
\begin{aligned}
& \omega_{(M)}+a=\frac{1}{2} \frac{\partial_{a} \eta}{1-\eta}, \quad \omega_{\mu \underset{(A)(A)}{+}}=-\frac{1}{2} \delta_{\mu i} \frac{\partial_{i} \eta}{1-\eta}, \quad \omega_{\mu-a}=\frac{1}{2} \delta_{\mu+} \frac{\partial_{a} \eta}{(1-\eta)^{2}}, \\
& \Gamma_{\mu-}^{-}=\frac{1}{2} \delta_{\mu i} \frac{\partial_{i} \eta}{1-\eta}, \quad \Gamma_{\mu-}^{\rho}=-\frac{1}{2} \delta_{\mu+} \delta^{\rho i} \frac{\partial_{i} \eta}{(1-\eta)^{2}}, \quad \Gamma_{\mu \nu}^{\rho}=\frac{1}{2} \delta^{\rho+} \delta_{\mu+} \delta_{\nu i} \frac{\partial_{i} \eta}{1-\eta} .
\end{aligned}
$$

The imaginary time version of operator $\hat{M}_{\hat{\mathcal{O}}}$ is obtained by using eq. (4.6):

$$
\begin{aligned}
& g^{1 / 4}\left(\square-\frac{1}{4} R\right)_{E} g^{-1 / 4} \Psi= \\
& =\left[-2 i m \mathbf{1}+2 i m \eta \mathbf{1}+\frac{i}{2}\left(\partial_{a} \eta\right) \gamma^{+a}\right] \partial_{t} \Psi+\left[-\frac{1}{2}\left(\partial_{a} \eta\right) \gamma^{-+}-\frac{1}{2} \eta\left(\partial_{a} \eta\right) \gamma^{-+}\right] \partial_{a} \Psi \\
& \quad+\left[\frac{1}{8}\left(\partial_{a} \eta\right)^{2} \mathbf{1}-\frac{1}{4} \partial^{2} \eta \gamma^{-+}-\frac{1}{4} \eta\left(\partial^{2} \eta\right) \gamma^{-+}-\frac{1}{4}\left(\partial_{a} \eta\right)^{2} \gamma^{-+}-\frac{1}{2} m\left(\partial_{a} \eta\right) \gamma^{-a}-\frac{1}{2} m \eta\left(\partial_{a} \eta\right) \gamma^{-a}\right] \Psi \\
& \quad+\left[\frac{1}{16}\left(\partial_{a} \eta\right)^{2} \mathbf{1}+\frac{1}{16}\left(\partial_{a} \eta\right)\left(\partial_{b} \eta\right)\left\{\gamma^{-a}, \gamma^{+b}\right\}\right] \Psi+\partial^{2} \Psi
\end{aligned}
$$

We will need the matrix elements of $\hat{M}$ in coordinate representation; to this purpose, it is useful to use the following decomposition:

$$
\begin{aligned}
\left\langle x t|\hat{M}| x^{\prime} t^{\prime}\right\rangle=\langle x t|[\triangle \mathbf{1} & +P(x) \delta\left(x-x^{\prime}\right) \delta\left(t-t^{\prime}\right)+S(x) \sqrt{-\partial_{t}^{2}} \delta\left(x-x^{\prime}\right) \delta\left(t-t^{\prime}\right) \\
& \left.+a_{i}(x) \partial_{i} \delta\left(x-x^{\prime}\right) \delta\left(t-t^{\prime}\right)\right]\left|x^{\prime} t^{\prime}\right\rangle
\end{aligned}
$$

where

$$
\begin{aligned}
P(x)= & \frac{3}{16}\left(\partial_{i} \eta\right)^{2} \mathbf{1}-\frac{1}{4}\left(\partial^{2} \eta\right) \gamma^{-+}-\frac{1}{4} \eta\left(\partial^{2} \eta\right) \gamma^{-+}-\frac{1}{4}\left(\partial_{i} \eta\right)^{2} \gamma^{-+} \\
& -\frac{1}{2} m\left(\partial_{i} \eta\right) \gamma^{-i}-\frac{1}{2} m \eta\left(\partial_{i} \eta\right) \gamma^{-i}+\frac{1}{16}\left(\partial_{i} \eta\right)\left(\partial_{j} \eta\right)\left\{\gamma^{-i}, \gamma^{-j}\right\} \\
S(x)= & 2 m \eta \mathbf{1}+\frac{1}{2}\left(\partial_{i} \eta\right) \gamma^{+i} \\
a_{i}(x)= & -\frac{1}{2}\left(\partial_{i} \eta\right) \gamma^{-+}-\frac{1}{2} \eta\left(\partial_{i} \eta\right) \gamma^{-+}
\end{aligned}
$$


A more explicit form is:

$$
P(x)=\left(\begin{array}{cccc}
P_{11}(x) & 0 & 0 & 0 \\
P_{21}(x) & P_{22}(x) & 0 & 0 \\
0 & 0 & P_{22}(x) & P_{32}(x) \\
0 & 0 & 0 & P_{11}(x)
\end{array}\right)
$$

where

$$
\begin{aligned}
& P_{11}(x)=\frac{5}{16}\left(\partial_{i} \eta\right)^{2}+\frac{1}{4}\left(\partial^{2} \eta\right)+\frac{1}{4} \eta\left(\partial^{2} \eta\right), \\
& P_{22}(x)=-\frac{3}{16}\left(\partial_{i} \eta\right)^{2}-\frac{1}{4}\left(\partial^{2} \eta\right)-\frac{1}{4} \eta\left(\partial^{2} \eta\right), \\
& P_{21}(x)=\frac{\sqrt{2}}{2} m\left[\left(\partial_{1}+i \partial_{2}\right) \eta+\eta\left(\partial_{1}+i \partial_{2}\right) \eta\right], \\
& P_{32}(x)=\frac{\sqrt{2}}{2} m\left[\left(-\partial_{1}+i \partial_{2}\right) \eta+\eta\left(-\partial_{1}+i \partial_{2}\right) \eta\right] .
\end{aligned}
$$

Moreover:

$$
S(x)=\left(\begin{array}{cccc}
S_{11}(x) & S_{12}(x) & 0 & 0 \\
0 & S_{11}(x) & 0 & 0 \\
0 & 0 & S_{11}(x) & 0 \\
0 & 0 & S_{43}(x) & S_{11}(x)
\end{array}\right)
$$

where

$$
S_{11}(x)=2 m \eta, \quad S_{12}(x)=\frac{\sqrt{2}}{2}\left(\partial_{1}-i \partial_{2}\right) \eta, \quad S_{43}(x)=-\frac{\sqrt{2}}{2}\left(\partial_{1}+i \partial_{2}\right) \eta,
$$

and:

$$
a_{i}(x)=a_{11}(x)\left(\begin{array}{cccc}
1 & 0 & 0 & 0 \\
0 & -1 & 0 & 0 \\
0 & 0 & -1 & 0 \\
0 & 0 & 0 & 1
\end{array}\right), \quad a_{11}(x)=\frac{1}{2}\left(\partial_{i} \eta\right)+\frac{1}{2} \eta\left(\partial_{i} \eta\right) .
$$

\subsection{Perturbative expansion}

The next task is to obtain the De Witt-Seeley-Gilkey expansion of the HK operator, in order to find the $a_{4}$ coefficient and then the trace anomaly. We will split $\hat{M}$ in a free part plus a perturbation $\hat{V}$ :

$$
\left\langle x t|\hat{M}| x^{\prime} t^{\prime}\right\rangle=\left\langle x t|\triangle \mathbf{1}+\hat{V}| x^{\prime} t^{\prime}\right\rangle=g^{1 / 4}(\triangle \mathbf{1}+\delta \triangle)\left[g^{-1 / 4} \delta\left(x-x^{\prime}\right) \delta\left(t-t^{\prime}\right)\right] .
$$

We can expand perturbatively the HK as a Dyson series:

$$
\hat{K}_{\hat{M}}(s)=\exp [s(\triangle \mathbf{1}+\hat{V})]=\sum_{n=0}^{\infty} \hat{K}_{n}(s)
$$

where the terms of the sum are

$$
\hat{K}_{n}(s)=\int_{0}^{s} d s_{n} \int_{0}^{s_{n}} d s_{n-1} \ldots \int_{0}^{s_{2}} d s_{1} e^{\left(s-s_{n}\right) \triangle \mathbf{1}} \hat{V} e^{\left(s_{n}-s_{n-1}\right) \triangle \mathbf{1}} \hat{V} \ldots e^{\left(s_{2}-s_{1}\right) \triangle \mathbf{1}} \hat{V} e^{s_{1} \Delta \mathbf{1}} .
$$


Since we are perturbing around flat space, from [27] we have

$$
K_{\triangle}(s)=\left\langle x t\left|e^{s \triangle 1}\right| x^{\prime} t^{\prime}\right\rangle=\frac{1}{2 \pi} \frac{m s}{m^{2} s^{2}+\frac{1}{4}\left(t-t^{\prime}\right)^{2}} \frac{1}{(4 \pi s)^{d / 2}} \exp \left[-\frac{\left(x-x^{\prime}\right)^{2}}{4 s}\right] \mathbf{1},
$$

which gives

$$
\tilde{K}_{\triangle}(s)=\operatorname{Tr}\left\langle x t\left|e^{s \triangle \mathbf{1}}\right| x t\right\rangle=\frac{2}{m(4 \pi s)^{d / 2+1}} \operatorname{Tr}(\mathbf{1})=\frac{8}{m(4 \pi s)^{d / 2+1}} .
$$

\subsubsection{Single insertion}

At the first order the Dyson series is

$$
K_{1}(s)=\int_{0}^{s} d s^{\prime}\left\langle x t\left|e^{\left(s-s^{\prime}\right) \triangle} \hat{V} e^{s^{\prime} \triangle}\right| x^{\prime} t^{\prime}\right\rangle .
$$

According to eq. (4.23), we can decompose the expression as

$$
\begin{aligned}
K_{1}(s)=K_{1 P}(s)+K_{1 S}(s)+K_{1 a_{i}}(s)= & \operatorname{Tr} \int_{0}^{s} d s^{\prime}\left\langle x t\left|e^{\left(s-s^{\prime}\right) \triangle} P(x) e^{s^{\prime} \triangle}\right| x^{\prime} t^{\prime}\right\rangle \\
& +\operatorname{Tr} \int_{0}^{s} d s^{\prime}\left\langle x t\left|e^{\left(s-s^{\prime}\right) \triangle} S(x) \sqrt{-\partial_{t}^{2}} e^{s^{\prime} \triangle}\right| x^{\prime} t^{\prime}\right\rangle \\
& +\operatorname{Tr} \int_{0}^{s} d s^{\prime}\left\langle x t\left|e^{\left(s-s^{\prime}\right) \triangle} a_{i}(x) \partial_{i} e^{s^{\prime} \triangle}\right| x^{\prime} t^{\prime}\right\rangle .
\end{aligned}
$$

The contribution $K_{1 a_{i}}(s)$ contains an implicit sum over the index $i$.

Note that we also introduced in the expression the trace operation, since we are now dealing with squared matrices and the heat kernel expansion required a trace over the operator considered.

We can use the following results from appendix A of [27]:

$$
\begin{aligned}
& \tilde{K}_{1 P}=\frac{2}{m(4 \pi s)^{d / 2+1}} \operatorname{Tr}\left(s P+\frac{1}{6} s^{2} \partial_{x}^{2} P+\ldots\right) \\
& \tilde{K}_{1 S}=\frac{2}{m(4 \pi s)^{d / 2+1}} \operatorname{Tr}\left(\frac{S}{2 m}+\frac{s}{12 m} \partial^{2} S+\frac{s^{2}}{120 m} \partial^{4} S+\ldots\right) .
\end{aligned}
$$

Moreover the contribution $\tilde{K}_{1 a_{i}}$ due to $a_{i}$ is the sum of the trace of various terms proportional to the derivatives of $a_{i}$; these terms have all zero trace and so $\tilde{K}_{1 a_{i}}=0$.

\subsubsection{Double insertion}

At the second order the heat kernel expansion is

$$
K_{2}(s)=\int_{0}^{s} d s^{\prime} \int_{0}^{s^{\prime}} d s^{\prime \prime}\left\langle x t\left|e^{\left(s-s^{\prime}\right) \triangle} \hat{V} e^{\left(s^{\prime}-s^{\prime \prime}\right) \triangle} \hat{V} e^{s^{\prime \prime} \triangle}\right| x^{\prime} t^{\prime}\right\rangle .
$$

$K_{2}$ splits into the sum of several contributions:

$$
\begin{aligned}
K_{2}(s)=\sum_{X} K_{2 X}(s)= & K_{2 P P}(s)+K_{2 S S}(s)+K_{2 P S}(s)+K_{2 S P}(s)+K_{2 a_{i} a_{j}}(s) \\
& +K_{2 a_{i} P}(s)+K_{2 P a_{i}}(s)+K_{2 a_{i} S}(s)+K_{2 S a_{i}}(s),
\end{aligned}
$$

where in each contribution there is an implicit sum over the indices $i, j$. 
We can use the following results from appendix B of [27]:

$$
\begin{aligned}
\tilde{K}_{2 P P}= & \frac{2}{m(4 \pi s)^{d / 2+1}} \operatorname{Tr}\left(\frac{s^{2}}{2} P(x)^{2}+\ldots\right) \\
\tilde{K}_{2 S S}= & \frac{2}{m(4 \pi s)^{d / 2+1}} \operatorname{Tr}\left(\frac{S^{2}}{4 m^{2}}+\frac{s}{12 m^{2}} S \partial^{2} S+\frac{s}{24 m^{2}} \partial_{k} S \partial_{k} S+\frac{s^{2}}{120 m^{2}} S \partial^{4} S\right. \\
& \left.+\frac{s^{2}}{144 m^{2}} \partial^{2} S \partial^{2} S+\frac{s^{2}}{60 m^{2}} \partial_{i} \partial^{2} S \partial_{i} S+\frac{s^{2}}{180 m^{2}} \partial_{i j} S \partial_{i j} S+\ldots\right), \\
\tilde{K}_{2 P S}=\tilde{K}_{2 S P}= & \frac{1}{m(4 \pi s)^{d / 2+1}} \operatorname{Tr}\left(\frac{s}{2 m} S P+\frac{s^{2}}{12 m} S \partial^{2} P+\frac{s^{2}}{12 m} \partial^{2} S P+\frac{s^{2}}{12 m} \partial_{i} S \partial_{i} P+\ldots\right) .
\end{aligned}
$$

For the remaining terms, the calculation is performed in appendix C:

$$
\begin{aligned}
\tilde{K}_{2 a_{j} a_{i}}= & \frac{2}{m(4 \pi s)^{d / 2+1}} \operatorname{Tr}\left[-\frac{s}{4} a_{i} a_{i}-\frac{s^{2}}{24}\left(\partial_{j} a_{i}\right)\left(\partial_{i} a_{j}\right)\right. \\
& \left.+\frac{s^{2}}{8}\left(\partial_{i} a_{i}\right)\left(\partial_{j} a_{j}\right)-\frac{s^{2}}{12} a_{i}\left(\partial^{2} a_{i}\right)-\frac{s^{2}}{24}\left(\partial_{i} a_{j}\right)^{2}+\ldots\right], \\
\tilde{K}_{2 a_{i} P}= & \frac{2}{m(4 \pi s)^{d / 2+1}} \operatorname{Tr}\left(-\frac{s^{2}}{3} P\left(\partial_{i} a_{i}\right)-\frac{s^{2}}{6}\left(\partial_{i} P\right) a_{i}+\ldots\right), \\
\tilde{K}_{2 P a_{i}}= & \frac{2}{m(4 \pi s)^{d / 2+1}} \operatorname{Tr}\left(\frac{s^{2}}{6} a_{i}\left(\partial_{i} P\right)-\frac{s^{2}}{6}\left(\partial_{i} a_{i}\right) P+\ldots\right) .
\end{aligned}
$$

The expressions for $\tilde{K}_{2 a_{i} S}, \tilde{K}_{2 S a_{i}}$ involve traces of matrix products of the kind

$$
\operatorname{Tr} \partial^{k} a_{i}(x) \partial^{l} S(x),
$$

but these all vanish due to the structure of the matrices $a_{i}, S$, whose entries sit in orthogonal subspaces.

\subsubsection{Results}

Summing the contribution from the single and double insertions, we find $a_{4}$ up to the second order in $\eta$, for $d=2$ :

$$
\sqrt{g} a_{4}(\hat{\triangle})=\frac{2}{m(4 \pi)^{2}}\left[\frac{1}{15} \partial^{4} \eta+\frac{2}{15} \eta\left(\partial^{4} \eta\right)+\frac{13}{30}\left(\partial_{i} \eta\right)\left(\partial_{i} \partial^{2} \eta\right)+\frac{1}{9}\left(\partial^{2} \eta\right)^{2}+\frac{31}{180}\left(\partial_{i j} \eta\right)^{2}\right] .
$$

We should then express the result in terms of curvature invariants. Up to the second order in $\eta$, the curvature combinations entering the anomaly are given by:

$$
\begin{aligned}
\sqrt{g} D^{2} R & =-2 \partial^{4} \eta-4 \eta\left(\partial^{4} \eta\right)-13\left(\partial_{i} \eta\right)\left(\partial_{i} \partial^{2} \eta\right)-2\left(\partial^{2} \eta\right)^{2}-7\left(\partial_{i j} \eta\right)^{2} \\
\sqrt{g} E_{4} & =2\left(\partial^{2} \eta\right)^{2}-2\left(\partial_{i j} \eta\right)^{2}, \quad \sqrt{g} W^{2}=\frac{1}{3}\left(\partial^{2} \eta\right)^{2}
\end{aligned}
$$

In our conventions the Euler density $E_{4}$ and the square of the Weyl tensor $W^{2}$ are, in term of the Riemann and Ricci tensor of the null reduction metric eq. (2.4):

$$
E_{4}=R_{A B M N}^{2}-4 R_{A B}^{2}+R^{2}, \quad W_{A B M N}^{2}=R_{A B M N}^{2}-2 R_{A B}^{2}+\frac{1}{3} R^{2} .
$$


Since we are studying a Weyl-invariant operator, we know from the Wess-Zumino consistency conditions that the $R^{2}$ term cannot enter the anomaly. We can then write the result as:

$$
a_{4}\left(\not D_{E}^{2}\right)=\frac{1}{8 m \pi^{2}}\left(\frac{11}{360} E_{4}-\frac{1}{20} W^{2}-\frac{1}{30} D^{2} R\right)
$$

The trace anomaly then can be computed as follows:

$$
\mathcal{A}=-\frac{1}{2} a_{4}\left(\not D_{E}^{2}\right)
$$

\section{Conclusions}

In this paper we checked that the action of a non-relativistic spin $1 / 2$ fermion coupled to NC geometry is Weyl invariant. Then the trace anomaly was computed using the HK method; the result is for a fermionic spin doublet:

$$
\mathcal{A}=T_{i}^{i}-2 T_{0}^{0}=\left(-a E_{4}+c W^{2}+b R^{2}+a^{\prime} D^{2} R\right)+\ldots .
$$

where

$$
a=\frac{1}{8 m \pi^{2}} \frac{1}{360} \frac{11}{2}, \quad c=\frac{1}{8 m \pi^{2}} \frac{9}{360}, \quad b=0, \quad a^{\prime}=\frac{1}{8 m \pi^{2}} \frac{6}{360},
$$

and the dots stand for possible additional terms, both higher derivatives and of the kind discussed in [28], which violate the Milne boost symmetry.

Up to an overall $1 / m$ multiplicative factor, the anomaly coefficients turn out to be proportional to the ones of a relativistic Dirac fermion in 4 dimensions. A similar numerical coincidence happens also in the scalar case [27], where the value of the anomaly coefficients is:

$$
a=\frac{1}{8 m \pi^{2}} \frac{1}{360}, \quad c=\frac{1}{8 m \pi^{2}} \frac{3}{360}, \quad b=\frac{1}{8 m \pi^{2}} \frac{1}{2}\left(\xi-\frac{1}{6}\right)^{2}, \quad a^{\prime}=\frac{1}{8 m \pi^{2}} \frac{1-5 \xi}{30},
$$

where $\xi$ is the parameter multiplying the conformal coupling.

It is natural to conjecture that an analog of the $a$-theorem may hold for the $E_{4}$ coefficient of Schrödinger-invariant theories in $2+1$ dimensions. For example, in the case where both the elementary and the composite degrees of freedom would be free scalars and fermions with spin $1 / 2$, it would imply that

$$
a_{\mathrm{UV}} \propto \sum_{\text {scalars }}^{\mathrm{UV}} \frac{1}{m}+\frac{11}{2} \sum_{\text {fermions }}^{\mathrm{UV}} \frac{1}{m} \geq \sum_{\text {scalars }}^{\mathrm{IR}} \frac{1}{m}+\frac{11}{2} \sum_{\text {fermions }}^{\mathrm{IR}} \frac{1}{m} \propto a_{\mathrm{IR}} .
$$

In Galilean-invariant theories the mass is a conserved quantity and the mass of a bound state is equal to the sum of the masses of the elementary constituents: no bound-state contribution to the mass is present as in the relativistic case. As proposed in [27], the $1 / m$ dependence is consistent with the intuition that bound states form in the infrared: as energy is added bound states tend to be broken.

Several interesting problems require further investigation: 
- Some new anomaly terms were computed in [28] using Fujikawa approach; they are present when a non-trivial background $\mathrm{U}(1)$ gauge field is added and they violate Milne boost symmetry. Wess-Zumino consistency conditions for these new terms should be studied and the computation should be checked using HK method.

- The relation between the anomaly coefficients and the correlation functions of the energy-momentum tensor multiplet should be clarified. In the case of vacuum correlation function, these correlators have support just at coincident points. It would be interesting to check if the anomaly coefficients can be related to the form of the finite-density correlators evaluated at separated points.

- The relation between the anomaly and the dilaton effective action should be investigated; in the relativistic case, this leads to a proof of the $a$-theorem [14]. The study of non-relativistic dilaton was initiated in [47].

- It would be interesting to attempt a perturbative proof using Osborn's local renormalization group approach; this was initiated in [26]. The main missing ingredient to the proof is to control the positivity of some anomaly coefficients whose relativistic analog turn out to be proportional to the Zamolodchikov metric. Local renormalization group for Lifshitz theories was studied in [48].

- In the relativistic supersymmetric case, there is a powerful relation between the trace anomaly coefficients and $R$-charges [49]; it would be interesting to check if a similar relation exists also in the non-relativistic case. The supersymmetric local RG approach as in [50] might be a convenient way to investigate these issues. NewtonCartan supergravity was studied in [51].

- The anomaly coefficients for anyons coupled to NC backgrounds should be computed. This may be interesting for condensed matter applications, as the quantum Hall effect.

\section{A Sigma and gamma matrices with light-cone indices}

We use the standard conventions:

$$
\sigma^{A}=\left(\mathbf{1}, \sigma^{\alpha}\right), \quad \bar{\sigma}^{A}=\left(-\mathbf{1}, \sigma^{\alpha}\right)
$$

It is useful to write the explicit expressions in lightcone indices:

- Sigma matrices in 4 dimensions

$$
\begin{aligned}
\sigma^{ \pm} & =\frac{1}{\sqrt{2}}\left(\sigma^{3} \pm \sigma^{0}\right), & \bar{\sigma}^{ \pm} & =\frac{1}{\sqrt{2}}\left(\bar{\sigma}^{3} \pm \bar{\sigma}^{0}\right), \\
\sigma^{-} & =\sqrt{2}\left(\begin{array}{cc}
0 & 0 \\
0 & -1
\end{array}\right), & \sigma^{+} & =\sqrt{2}\left(\begin{array}{cc}
1 & 0 \\
0 & 0
\end{array}\right), \\
\bar{\sigma}^{-} & =\sqrt{2}\left(\begin{array}{cc}
1 & 0 \\
0 & 0
\end{array}\right), & \bar{\sigma}^{+} & =\sqrt{2}\left(\begin{array}{cc}
0 & 0 \\
0 & -1
\end{array}\right), \\
\sigma^{1} & =\bar{\sigma}^{1}=\left(\begin{array}{cc}
0 & 1 \\
1 & 0
\end{array}\right), & \sigma^{2} & =\bar{\sigma}^{2}=\left(\begin{array}{cc}
0 & -i \\
i & 0
\end{array}\right),
\end{aligned}
$$


The Lorentz generators are then:

$$
\sigma^{A B}=\frac{1}{2}\left(\sigma^{A} \bar{\sigma}^{B}-\sigma^{B} \bar{\sigma}^{A}\right),
$$

which gives:

$$
\begin{array}{rlrl}
\sigma^{-1} & =-\frac{1}{\sqrt{2}}\left(\sigma^{1}-i \sigma^{2}\right)=\sqrt{2}\left(\begin{array}{cc}
0 & 0 \\
-1 & 0
\end{array}\right), & \sigma^{-2}=-\frac{1}{\sqrt{2}}\left(\sigma^{2}+i \sigma^{1}\right)=\sqrt{2} i\left(\begin{array}{cc}
0 & 0 \\
-1 & 0
\end{array}\right), \\
\sigma^{+1}=\frac{1}{\sqrt{2}}\left(\sigma^{1}+i \sigma^{2}\right)=\sqrt{2}\left(\begin{array}{cc}
0 & 1 \\
0 & 0
\end{array}\right), & \sigma^{+2}=-\frac{1}{\sqrt{2}}\left(i \sigma^{1}-\sigma^{2}\right)=\sqrt{2} i\left(\begin{array}{cc}
0 & -1 \\
0 & 0
\end{array}\right), \\
\sigma^{-+}=-\sigma^{3}=\left(\begin{array}{cc}
-1 & 0 \\
0 & 1
\end{array}\right), & \sigma^{12}=i \sigma^{3}=\left(\begin{array}{cc}
i & 0 \\
0 & -i
\end{array}\right) .
\end{array}
$$

- Gamma matrices in 4 dimensions

$$
\begin{array}{ll}
\gamma^{-}=\frac{1}{\sqrt{2}}\left(\gamma^{3}-\gamma^{0}\right)=\sqrt{2}\left(\begin{array}{cccc}
0 & 0 & 0 & 0 \\
0 & 0 & 0 & -1 \\
1 & 0 & 0 & 0 \\
0 & 0 & 0 & 0
\end{array}\right), & \gamma^{+}=\frac{1}{\sqrt{2}}\left(\gamma^{3}+\gamma^{0}\right)=\sqrt{2}\left(\begin{array}{cccc}
0 & 0 & 1 & 0 \\
0 & 0 & 0 & 0 \\
0 & 0 & 0 & 0 \\
0 & -1 & 0 & 0
\end{array}\right), \\
\gamma^{1}=\left(\begin{array}{cc}
0 & \sigma^{1} \\
\sigma^{1} & 0
\end{array}\right), & \gamma^{2}=\left(\begin{array}{cc}
0 & \sigma^{2} \\
\sigma^{2} & 0
\end{array}\right),
\end{array}
$$

The Lorentz generators are:

$$
\gamma^{A B}=\frac{1}{2}\left[\gamma^{A}, \gamma^{B}\right]
$$

which gives:

$$
\begin{aligned}
\gamma^{-+}=\left(\begin{array}{cccc}
-1 & 0 & 0 & 0 \\
0 & 1 & 0 & 0 \\
0 & 0 & 1 & 0 \\
0 & 0 & 0 & -1
\end{array}\right), & \gamma^{-1}=\left(\begin{array}{cccc}
0 & 0 & 0 & 0 \\
-\sqrt{2} & 0 & 0 & 0 \\
0 & 0 & 0 & \sqrt{2} \\
0 & 0 & 0 & 0
\end{array}\right), \\
\gamma^{+1}=\left(\begin{array}{cccc}
0 & \sqrt{2} & 0 & 0 \\
0 & 0 & 0 & 0 \\
0 & 0 & 0 & 0 \\
0 & 0 & -\sqrt{2} & 0
\end{array}\right), & \gamma^{+2}=\left(\begin{array}{cccc}
0-\sqrt{2} i & 0 & 0 \\
0 & 0 & 0 & 0 \\
0 & 0 & 0 & 0 \\
0 & 0 & -\sqrt{2} i & 0
\end{array}\right) .
\end{aligned}
$$

\section{B Spin connection}

The explicit expression for the spin connection is:

$$
\begin{gathered}
\omega_{M A B}=\frac{1}{2}\left[e_{A}^{N}\left(\partial_{M} e_{N B}-\partial_{N} e_{M B}\right)-e_{B}^{N}\left(\partial_{M} e_{N A}-\partial_{N} e_{M A}\right)\right. \\
\left.-e^{N}{ }_{A} e_{B}^{P}\left(\partial_{N} e_{P C}-\partial_{P} e_{N C}\right) e^{C}{ }_{M}\right] .
\end{gathered}
$$


We thus obtain the components:

$$
\begin{aligned}
\omega_{(M)}{ }_{(M B}= & -\frac{1}{2} e^{\mu}{ }_{A} e^{\nu}{ }_{B} \tilde{F}_{\mu \nu}, \quad \omega_{\mu-A}=-\frac{1}{2} e^{\nu}{ }_{A} \tilde{F}_{\mu \nu}, \\
\omega_{\mu+a}= & \frac{1}{2} v^{\nu}\left(\partial_{\mu} e^{a}{ }_{\nu}-\partial_{\nu} e^{a}{ }_{\mu}\right)-\frac{1}{2} e^{\nu}{ }_{a} F_{\mu \nu} \\
& -\frac{1}{2} v^{\nu} e^{\rho}{ }_{a}\left[A_{\mu} \tilde{F}_{\nu \rho}+n_{\mu} F_{\nu \rho}+e^{b}{ }_{\mu}\left(\partial_{\nu} e^{b}{ }_{\rho}-\partial_{\rho} e^{b}{ }_{\nu}\right)\right], \\
\omega_{\mu a b}= & \frac{1}{2} e^{\nu}{ }_{a}\left(\partial_{\mu} e^{b}{ }_{\nu}-\partial_{\nu} e^{b}{ }_{\mu}\right)-\frac{1}{2} e^{\nu}{ }_{b}\left(\partial_{\mu} e^{a}{ }_{\nu}-\partial_{\nu} e^{a}{ }_{\mu}\right) \\
& -\frac{1}{2} e^{\nu}{ }_{a} e^{\rho}{ }_{b}\left[A_{\mu} \tilde{F}_{\nu \rho}+n_{\mu} F_{\nu \rho}+e_{\mu}^{c}\left(\partial_{\nu} e_{\rho}^{c}-\partial_{\rho} e^{c}{ }_{\nu}\right)\right] .
\end{aligned}
$$

Note that $\omega_{(M)}-B=0$.

\section{Some double insertion contributions to the Heat Kernel}

Here we consider contributions of the form $K_{2 X_{1} X_{2}}(s)$, where

$$
X_{1}=\left\{P\left(x_{1}\right), a_{i}\left(x_{1}\right)\right\}, \quad X_{2}=\left\{P\left(x_{2}\right), a_{j}\left(x_{2}\right)\right\}
$$

whose explicit expression is:

$$
\begin{aligned}
K_{2 X_{1} X_{2}}(s)= & \int_{0}^{s} d s_{2} \int_{0}^{s_{2}} d s_{1} \\
& \left\langle x^{\prime} t^{\prime}\left|e^{-\left(s-s_{2}\right) \triangle}\right| x_{2} t_{2}\right\rangle \hat{X}_{2}\left\langle x_{2} t_{2}\left|e^{-\left(s_{2}-s_{1}\right)}\right| x_{1} t_{1}\right\rangle \hat{X}_{1}\left\langle x_{1} t_{1}\left|e^{-s_{1} \Delta}\right| x t\right\rangle,
\end{aligned}
$$

where

$$
\hat{X}_{1}=\left\{P\left(x_{1}\right), a_{i}\left(x_{1}\right) \partial_{x_{1}, i}\right\}, \quad \hat{X}_{2}=\left\{P\left(x_{2}\right), a_{j}\left(x_{2}\right) \partial_{x_{2}, j}\right\} .
$$

The quantity $K_{2 P P}$ was already computed in [27].

We can split the integration as follows:

$$
K_{2 X_{1} X_{2}}(s)=\int_{0}^{s} d s_{2} \int_{0}^{s_{2}} d s_{1} \frac{1}{\left(4 \pi\left(s-s_{2}\right)\right)^{d / 2}} \frac{1}{\left(4 \pi\left(s_{2}-s_{1}\right)\right)^{d / 2}} \frac{1}{\left(4 \pi s_{1}\right)^{d / 2}} \Xi^{X_{1} X_{2}} \Theta
$$

where $\Xi^{X_{1} X_{2}}$ and $\Theta$ correspond to the space and time integrals, respectively. It is useful to Fourier transform:

$$
\Xi^{X_{1} X_{2}}=\int \frac{d^{d} k_{1}}{(2 \pi)^{d / 2}} \frac{d^{d} k_{2}}{(2 \pi)^{d / 2}} \tilde{\Xi}^{X_{1} X_{2}}
$$

and to introduce:

$$
\Upsilon=\exp \left(i k_{1} x_{1}+i k_{2} x_{2}-\frac{\left(x^{\prime}-x_{2}\right)^{2}}{4\left(s-s_{2}\right)}-\frac{\left(x_{2}-x_{1}\right)^{2}}{4\left(s_{2}-s_{1}\right)}-\frac{\left(x_{1}-x\right)^{2}}{4 s_{1}}\right) .
$$


The Fourier transforms of the space part of the integrals are:

$$
\begin{aligned}
\tilde{\Xi}^{P P} & =\int d x_{1} \int d x_{2} \Upsilon P\left(k_{1}\right) P\left(k_{2}\right), \\
\tilde{\Xi}^{a_{i} P} & =-\partial_{x, i}\left[\int d x_{1} \int d x_{2} \Upsilon a_{i}\left(k_{1}\right) P\left(k_{2}\right)\right], \\
\tilde{\Xi}^{P a_{j}} & =\int d x_{1} \int d x_{2}\left[-\frac{\left(x_{2}-x_{1}\right)_{j}}{2\left(s_{2}-s_{1}\right)}\right] \Upsilon P\left(k_{1}\right) a_{j}\left(k_{2}\right), \\
\tilde{\Xi}^{a_{i} a_{j}} & =-\partial_{x, i}\left[\int d x_{1} \int d x_{2}\left[-\frac{\left(x_{2}-x_{1}\right)_{j}}{2\left(s_{2}-s_{1}\right)}\right] \Upsilon a_{i}\left(k_{1}\right) a_{j}\left(k_{2}\right)\right],
\end{aligned}
$$

where $P(k)$ and $a_{i}(k)$ are the Fourier transform of $P(x)$ and $a_{i}(x)$. The two basic integrals give:

$$
\begin{aligned}
\tilde{\Xi}^{P P}= & (4 \pi)^{d}\left(\frac{s_{1}\left(s-s_{2}\right)\left(s_{2}-s_{1}\right)}{s}\right)^{d / 2} \\
& \times \exp \left(\frac{i k_{1} s_{1} x^{\prime}}{s}+\frac{i k_{2} s_{2} x^{\prime}}{s}-\frac{i k_{1} s_{1} x}{s}-\frac{i k_{2} s_{2} x}{s}+\frac{k_{1}^{2} s_{1}^{2}}{s}+\frac{k_{2}^{2} s_{2}^{2}}{s}-k_{1}^{2} s_{1}\right. \\
& \left.-2 k_{1} k_{2} s_{1}-k_{2}^{2} s_{2}+\frac{2 k_{1} k_{2} s_{1} s_{2}}{s}+i k_{1} x+i k_{2} x-\frac{x^{2}}{4 s}+\frac{x x^{\prime}}{2 s}-\frac{\left(x^{\prime}\right)^{2}}{4 s}\right) P\left(k_{1}\right) P\left(k_{2}\right), \\
\tilde{\Xi}^{P a_{j}}= & \exp \left(\frac{i k_{1} s_{1} x^{\prime}}{s}+\frac{i k_{2} s_{2} x^{\prime}}{s}-\frac{i k_{1} s_{1} x}{s}-\frac{i k_{2} s_{2} x}{s}+\frac{k_{1}^{2} s_{1}^{2}}{s}+\frac{k_{2}^{2} s_{2}^{2}}{s}-k_{1}^{2} s_{1}\right. \\
& \left.-2 k_{1} k_{2} s_{1}-k_{2}^{2} s_{2}+\frac{2 k_{1} k_{2} s_{1} s_{2}}{s}+i k_{1} x+i k_{2} x+\frac{x x^{\prime}}{2 s}-\frac{\left(x^{\prime}\right)^{2}}{4 s}-\frac{x^{2}}{4 s}\right) \\
& \times(4 \pi)^{d}\left(\frac{s_{1}\left(s_{1}-s_{2}\right)\left(s_{2}-s\right)}{s}\right)^{d / 2} \frac{\left(i k_{1} s_{1}+i k_{2} s-i k_{2} s+\frac{x-x^{\prime}}{2}\right)_{i}}{s} P\left(k_{1}\right) a_{j}\left(k_{2}\right) .
\end{aligned}
$$

The expressions for $\tilde{\Xi}^{a_{i} P}$ and $\tilde{\Xi}^{a_{i} a_{j}}$ can be obtained differentiating $\tilde{\Xi}^{P P}$ and $\tilde{\Xi}^{P a_{j}}$ with respect to $x_{i}$. The time part gives:

$$
\begin{aligned}
\Theta & =\frac{1}{(2 \pi)^{3}} \int d t_{1} \int d t_{2} \frac{m\left(s-s_{2}\right)}{m^{2}\left(s-s_{2}\right)^{2}+\frac{\left(t_{2}-t^{\prime}\right)^{2}}{4}} \frac{m\left(s_{2}-s_{1}\right)}{m^{2}\left(s_{2}-s_{1}\right)^{2}+\frac{\left(t_{2}-t_{1}\right)^{2}}{4}} \frac{m s_{1}}{m^{2} s_{1}^{2}+\frac{\left(t_{1}-t\right)^{2}}{4}} \\
& =\frac{1}{\pi} \frac{2 m s}{4 m^{2} s^{2}+\left(t-t^{\prime}\right)^{2}} .
\end{aligned}
$$

Combining all the expressions and specializing to $x=x^{\prime}, t=t^{\prime}$, we find eqs. (4.44)-(4.46).

Open Access. This article is distributed under the terms of the Creative Commons Attribution License (CC-BY 4.0), which permits any use, distribution and reproduction in any medium, provided the original author(s) and source are credited.

\section{References}

[1] C.W. Misner, K.S. Thorne and J.A. Wheeler, Gravitation, W.H. Freeman, San Francisco, U.S.A. (1973). 
[2] D.T. Son and M. Wingate, General coordinate invariance and conformal invariance in nonrelativistic physics: Unitary Fermi gas, Annals Phys. 321 (2006) 197 [cond-mat/0509786] [INSPIRE].

[3] C. Hoyos and D.T. Son, Hall viscosity and electromagnetic response, Phys. Rev. Lett. 108 (2012) 066805 [arXiv:1109.2651] [INSPIRE].

[4] D.T. Son, Newton-Cartan geometry and the quantum Hall effect, arXiv:1306.0638 [INSPIRE].

[5] M. Geracie, D.T. Son, C. Wu and S.-F. Wu, Spacetime symmetries of the quantum Hall effect, Phys. Rev. D 91 (2015) 045030 [arXiv: 1407.1252] [InSPIRE].

[6] A.B. Zamolodchikov, Irreversibility of the flux of the renormalization group in a $2 D$ field theory, JETP Lett. 43 (1986) 730 [Pisma Zh. Eksp. Teor. Fiz. 43 (1986) 565] [INSPIRE].

[7] D.L. Jafferis, The exact superconformal R-symmetry extremizes Z, JHEP 05 (2012) 159 [arXiv: 1012.3210] [INSPIRE].

[8] D.L. Jafferis, I.R. Klebanov, S.S. Pufu and B.R. Safdi, Towards the F-theorem: $N=2$ field theories on the three-sphere, JHEP 06 (2011) 102 [arXiv:1103.1181] [INSPIRE].

[9] S.S. Pufu, The F-theorem and F-maximization, arXiv:1608.02960 [INSPIRE].

[10] J.L. Cardy, Is there a c theorem in four-dimensions?, Phys. Lett. B 215 (1988) 749 [INSPIRE].

[11] H. Osborn, Derivation of a four-dimensional c theorem, Phys. Lett. B 222 (1989) 97 [INSPIRE].

[12] I. Jack and H. Osborn, Analogs for the c theorem for four-dimensional renormalizable field theories, Nucl. Phys. B 343 (1990) 647 [InSPIRE].

[13] H. Osborn, Weyl consistency conditions and a local renormalization group equation for general renormalizable field theories, Nucl. Phys. B 363 (1991) 486 [INSPIRE].

[14] Z. Komargodski and A. Schwimmer, On renormalization group flows in four dimensions, JHEP 12 (2011) 099 [arXiv: 1107.3987] [INSPIRE].

[15] Z. Komargodski, The constraints of conformal symmetry on RG flows, JHEP 07 (2012) 069 [arXiv:1112.4538] [INSPIRE].

[16] S. Deser and A. Schwimmer, Geometric classification of conformal anomalies in arbitrary dimensions, Phys. Lett. B 309 (1993) 279 [hep-th/9302047] [InSPIRE].

[17] I. Adam, I.V. Melnikov and S. Theisen, A non-relativistic Weyl anomaly, JHEP 09 (2009) 130 [arXiv:0907.2156] [INSPIRE].

[18] M. Baggio, J. de Boer and K. Holsheimer, Anomalous breaking of anisotropic scaling symmetry in the quantum Lifshitz model, JHEP 07 (2012) 099 [arXiv:1112.6416] [INSPIRE].

[19] T. Griffin, P. Hořava and C.M. Melby-Thompson, Conformal Lifshitz gravity from holography, JHEP 05 (2012) 010 [arXiv:1112.5660] [INSPIRE].

[20] I. Arav, S. Chapman and Y. Oz, Lifshitz scale anomalies, JHEP 02 (2015) 078 [arXiv: 1410.5831] [INSPIRE].

[21] I. Arav, S. Chapman and Y. Oz, Non-relativistic scale anomalies, JHEP 06 (2016) 158 [arXiv:1601.06795] [INSPIRE]. 
[22] A.O. Barvinsky et al., Heat kernel methods for Lifshitz theories, JHEP 06 (2017) 063 [arXiv: 1703.04747] [INSPIRE].

[23] I. Arav, Y. Oz and A. Raviv-Moshe, Lifshitz anomalies, Ward identities and split dimensional regularization, JHEP 03 (2017) 088 [arXiv:1612.03500] [INSPIRE].

[24] K. Jensen, Anomalies for Galilean fields, arXiv:1412.7750 [INSPIRE].

[25] R. Auzzi, S. Baiguera and G. Nardelli, On Newton-Cartan trace anomalies, JHEP 02 (2016) 003 [Erratum ibid. 02 (2016) 177] [arXiv:1511.08150] [INSPIRE].

[26] R. Auzzi, S. Baiguera, F. Filippini and G. Nardelli, On Newton-Cartan local renormalization group and anomalies, JHEP 11 (2016) 163 [arXiv:1610.00123] [INSPIRE].

[27] R. Auzzi and G. Nardelli, Heat kernel for Newton-Cartan trace anomalies, JHEP 07 (2016) 047 [arXiv: 1605. 08684] [INSPIRE].

[28] K. Fernandes and A. Mitra, Newton-Cartan gravitational anomalies of the Schrödinger field, arXiv: 1703.09162 [INSPIRE].

[29] S. Pal and B. Grinstein, On the heat kernel and Weyl anomaly of Schrödinger invariant theory, arXiv: 1703.02987 [INSPIRE].

[30] C. Duval, G. Burdet, H.P. Kunzle and M. Perrin, Bargmann structures and Newton-cartan theory, Phys. Rev. D 31 (1985) 1841 [INSPIRE].

[31] K. Jensen, On the coupling of Galilean-invariant field theories to curved spacetime, arXiv: 1408.6855 [INSPIRE].

[32] J. Hartong, E. Kiritsis and N.A. Obers, Schrödinger invariance from Lifshitz isometries in holography and field theory, Phys. Rev. D 92 (2015) 066003 [arXiv:1409.1522] [InSPIRE].

[33] J. Hartong, E. Kiritsis and N.A. Obers, Lifshitz space-times for Schrödinger holography, Phys. Lett. B 746 (2015) 318 [arXiv:1409.1519] [InSPIRE].

[34] J. Hartong, E. Kiritsis and N.A. Obers, Field theory on Newton-Cartan backgrounds and symmetries of the Lifshitz vacuum, JHEP 08 (2015) 006 [arXiv:1502.00228] [INSPIRE].

[35] M. Geracie, K. Prabhu and M.M. Roberts, Curved non-relativistic spacetimes, Newtonian gravitation and massive matter, J. Math. Phys. 56 (2015) 103505 [arXiv:1503.02682] [INSPIRE].

[36] M. Geracie, K. Prabhu and M.M. Roberts, Fields and fluids on curved non-relativistic spacetimes, JHEP 08 (2015) 042 [arXiv:1503.02680] [INSPIRE].

[37] M. Geracie, K. Prabhu and M.M. Roberts, Physical stress, mass and energy for non-relativistic matter, JHEP 06 (2017) 089 [arXiv: 1609.06729] [INSPIRE].

[38] M. Geracie, Galilean geometry in condensed matter systems, arXiv:1611.01198 [INSPIRE].

[39] R. Banerjee, A. Mitra and P. Mukherjee, Localization of the Galilean symmetry and dynamical realization of Newton-Cartan geometry, Class. Quant. Grav. 32 (2015) 045010 [arXiv: 1407.3617] [INSPIRE].

[40] R. Banerjee and P. Mukherjee, New approach to nonrelativistic diffeomorphism invariance and its applications, Phys. Rev. D 93 (2016) 085020 [arXiv:1509.05622] [INSPIRE].

[41] J.-M. Levy-Leblond, Nonrelativistic particles and wave equations, Commun. Math. Phys. 6 (1967) 286 [INSPIRE]. 
[42] J.F. Fuini, A. Karch and C.F. Uhlemann, Spinor fields in general Newton-Cartan backgrounds, Phys. Rev. D 92 (2015) 125036 [arXiv: 1510.03852] [InSPIRE].

[43] C. Duval, P.A. Horvathy and L. Palla, Spinors in nonrelativistic Chern-Simons electrodynamics, Annals Phys. 249 (1996) 265 [hep-th/9510114] [INSPIRE].

[44] S.M. Christensen and M.J. Duff, New gravitational index theorems and supertheorems, Nucl. Phys. B 154 (1979) 301 [INSPIRE].

[45] D.Z. Freedman and A. van Proyen, Supergravity, Cambridge University Press, Cambridge U.K. (2012).

[46] S.N. Solodukhin, Entanglement entropy in non-relativistic field theories, JHEP 04 (2010) 101 [arXiv:0909.0277] [INSPIRE].

[47] I. Arav, I. Hason and Y. Oz, Spontaneous breakingl of non-relativistic scale symmetry, arXiv: 1702.00690 [INSPIRE].

[48] S. Pal and B. Grinstein, Weyl consistency conditions in non-relativistic quantum field theory, JHEP 12 (2016) 012 [arXiv: 1605.02748] [INSPIRE].

[49] D. Anselmi, D.Z. Freedman, M.T. Grisaru and A.A. Johansen, Nonperturbative formulas for central functions of supersymmetric gauge theories, Nucl. Phys. B 526 (1998) 543 [hep-th/9708042] [INSPIRE].

[50] R. Auzzi and B. Keren-Zur, Superspace formulation of the local RG equation, JHEP 05 (2015) 150 [arXiv:1502.05962] [INSPIRE].

[51] R. Andringa, E.A. Bergshoeff, J. Rosseel and E. Sezgin, 3D Newton-Cartan supergravity, Class. Quant. Grav. 30 (2013) 205005 [arXiv:1305.6737] [INSPIRE]. 